\title{
The structure of communication problems in cellular automata
}

\author{
Raimundo Briceño ${ }^{1}$ \\ ${ }^{1}$ DIM, Universidad de Chile \\ ${ }^{2}$ LAMA, Université de Savoie, France
}

Pierre-Etienne Meunier ${ }^{21}$

Studying cellular automata with methods from communication complexity appears to be a promising approach. In the past, interesting connections between communication complexity and intrinsic universality in cellular automata were shown. One of the last extensions of this theory was its generalization to various "communication problems", or "questions" one might ask about the dynamics of cellular automata. In this article, we aim at structuring these problems, and find what makes them interesting for the study of intrinsic universality and quasi-orders induced by simulation relations.

Keywords: cellular automata, communication complexity, intrinsic universality, ideals

Outline. In Section 11 we recall the basic notions of communication complexity and its application to cellular automata. In Section 2, we show how communication complexity incorporates in the model of cellular automata, generalizing the previous works to other simulation relations, and developing new communication problems. Then, in Section 3 , we study sets of cellular automata closed under simulation (ideals), and how our communication approach relates with them.

\section{Introduction and definitions}

\subsection{Cellular automata and shift spaces}

In this paper we are always going to consider one-dimensional cellular automata (CA). A CA is defined by a local rule $\phi: Q^{2 r+1} \rightarrow Q$, where $r$ denotes the radius and $Q$, the set of states (or alphabet).

We denote by $\Phi: Q^{\mathbb{Z}} \rightarrow Q^{\mathbb{Z}}$ the global function induced by $\phi$ following the classical definition:

$$
\Phi(x)_{i}=\phi\left(x_{i-r}, \ldots, x_{i+r}\right),
$$

where $x$ is some element from $Q^{\mathbb{Z}}$ (or $Q_{\Phi}^{\mathbb{Z}}$, if we want to avoid ambiguities) called configuration. Finally, we denote by $\Phi^{t}$ the $t$-step iteration of the global function $\Phi$, such that $\Phi^{t+1}=\Phi^{t} \circ \Phi$ and $\Phi^{1}=\Phi$.

A global function $\Phi$ can be represented by different local rules. All properties considered in this paper depend only on $\Phi$ and are not sensitive to the choice of a particular local function. However, to avoid useless formalism, we will use the following notion of canonical local representation: $(\phi, r)$ is the canonical 
local representation of $\Phi$ if $\phi$ has radius $r$ and it is the local function of smallest radius having $\Phi$ as its associated global function. Throughout this work we are going to refer to a CA $\Phi$ with $(\phi, r)$.

The limit set of a given CA $\Phi$, denoted $\omega(\Phi)$, is defined as follows:

$$
\omega(\Phi)=\bigcap_{t \in \mathbb{N}} \Phi^{t}\left(Q^{\mathbb{Z}}\right)
$$

A limit set is always a non-empty shift space. A shift space $X$ over an alphabet $Q$ is any subset $X \subseteq Q^{\mathbb{Z}}$ that can be defined by a family of forbidden words $F \subseteq Q^{+}$, such that $X$ is the set of all configurations where no word of $F$ occurs. A shift space is said to be a shift of finite type (SFT) if it can be defined by a finite family $F$. We denote by $\mathcal{L}(X)$ the set of words occurring in configurations that belongs to $X$ and, by $\mathcal{L}_{n}(X):=\mathcal{L}(X) \cap Q^{n}$, its restriction to words of length $n \in \mathbb{N}$. A shift space is said to be a sofic shift if $\mathcal{L}(X)$ is a regular language. Clearly, every SFT is a sofic shift.

If there exists a time $t^{*} \in \mathbb{N}$ such that $\omega(\Phi)=\Phi^{t^{*}}\left(Q^{\mathbb{Z}}\right), \Phi$ is said to be stable, and unstable, otherwise. A stable limit set is always a sofic shift.

Finally, we denote $\mathcal{A C}$ the set of one-dimensional CAs.

\subsection{Simulations and universality}

We define two parallel notions of simulation between CAs developed in [DMOT11], based on geometrical transformations of diagram spaces and injections or projections between them.

Definition 1 (Rescaling) The ingredients of a rescaling are simple: packing cells into blocks, iterating the rule and composing with a traslation. Formally, given any state set $Q$ and any $m \geq 1$, we define the bijective packing map $b_{m}: Q^{\mathbb{Z}} \rightarrow\left(Q^{m}\right)^{\mathbb{Z}}$ by:

$$
\forall i \in \mathbb{Z}:\left(b_{m}(x)\right)(i)=(x(m i), \ldots, x(m i+m-1)),
$$

for all $x \in Q^{\mathbb{Z}}$. The rescaling $\Phi^{\langle m, t, z\rangle}$ of $\Phi$ by parameters $m$ (packing), $t \geq 1$ (iterating) and $z \in \mathbb{Z}$ (shifting, denoted $\sigma$ ) is the CA of state set $Q^{m}$ and global rule:

$$
b_{m} \circ \sigma^{z} \circ \Phi^{t} \circ b_{m}^{-1} .
$$

The fact that the above function is the global rule of a cellular automaton follows from Curtis-LyndonHedlund theorem [Hed69] because it is continuous and commutes with traslations.

In the rest of this section, we define various relations between cellular automata. They are all defined in [DMOT11], and we just recall them here.

Definition 2 (Sub-automaton) A CA $\Phi_{1}$ is a sub-automaton of a $C A \Phi_{2}$, denoted by $\Phi_{1} \sqsubseteq \Phi_{2}$, if there is an injective map $\iota$ from $Q_{1}$ to $Q_{2}$ such that $\bar{\iota} \circ \Phi_{1}=\Phi_{2} \circ \bar{\iota}$, where $\bar{\iota}: Q_{1}^{\mathbb{Z}} \rightarrow Q_{2}^{\mathbb{Z}}$ denotes the uniform extension of $\iota$.

Definition 3 (Quotient) A CA $\Phi_{1}$ is a quotient of a CA $\Phi_{2}$, denoted by $\Phi_{1} \unlhd \Phi_{2}$, if there is a surjective map $\varphi$ from $Q_{2}$ to $Q_{1}$ such that $\bar{\varphi} \circ \Phi_{2}=\Phi_{1} \circ \bar{\varphi}$, where $\bar{\varphi}: Q_{2}^{\mathbb{Z}} \rightarrow Q_{1}^{\mathbb{Z}}$ denotes the uniform extension of $\varphi$.

Definition 4 (Injective simulation) We say that $\Phi_{2}$ injectively simulates $\Phi_{1}$, denoted $\Phi_{1} \preccurlyeq{ }_{i} \Phi_{2}$, if there exist rescaling parameters $m_{1}, m_{2}, t_{1}, t_{2}, z_{1}$ and $z_{2}$ such that $\Phi_{1}{ }^{\left\langle m_{1}, t_{1}, z_{1}\right\rangle} \sqsubseteq \Phi_{2}{ }^{\left\langle m_{2}, t_{2}, z_{2}\right\rangle}$. 
Definition 5 (Surjective simulation) We say that $\Phi_{2}$ surjectively simulates $\Phi_{1}$, denoted $\Phi_{1} \preccurlyeq s \Phi_{2}$, if there exist rescaling parameters $m_{1}, m_{2}, t_{1}, t_{2}, z_{1}$ and $z_{2}$ such that $\Phi_{1}{ }^{\left\langle m_{1}, t_{1}, z_{1}\right\rangle} \unlhd \Phi_{2}{ }^{\left\langle m_{2}, t_{2}, z_{2}\right\rangle}$.

Definition 6 (Intrinsic universality) Let $\preccurlyeq \in\left\{\preccurlyeq_{i}, \preccurlyeq s\right\}$. $\Psi$ is intrinsically $\preccurlyeq$-universal if for all $\Phi$ it holds that $\Phi \preccurlyeq \Psi$.

It is well known that there exist intrinsically universal cellular automata for the $\preccurlyeq_{i}$ relation, and this property has been shown undecidable (see for instance [Oll03] and [DMOT11]). An open problem, appearing in various contexts (see [The05] or [BT10]), is the existence of a cellular automaton universal for the $\preccurlyeq_{s}$ relation:

Open Problem 1 Is there some $\Psi$ such that for all $\Phi$ it holds that $\Phi \preccurlyeq s \Psi$ ?

\subsection{Ideals}

Informally speaking, ideals are strict subsets of $\mathcal{A C}$ closed under simulation. In the general order theory, the precise definition is the following.

Definition 7 (Ideal) Let $\preccurlyeq$ be a quasiorder in $\mathcal{A C}$. An ideal $\mathcal{I}$ is a subset of $\mathcal{A C}$ such that:

1. If $\Phi_{2} \in \mathcal{I}$ and $\Phi_{1} \preccurlyeq \Phi_{2}$, then $\Phi_{1} \in \mathcal{I}$.

2. For any $\Phi_{1}, \Phi_{2} \in \mathcal{I}$ there is some $\Phi_{3} \in \mathcal{I}$ such that $\Phi_{1} \preccurlyeq \Phi_{3}$ and $\Phi_{2} \preccurlyeq \Phi_{3}$.

Moreover, $\mathcal{I}$ is said principal if there is some $\Phi_{I}$ such that:

$$
\Phi \in \mathcal{I} \Longleftrightarrow \Phi \preccurlyeq \Phi_{I} .
$$

Adapted to our context, we have the following sufficient conditions to be an ideal.

Proposition 1 ([DMOT11]) $\mathcal{I} \subseteq \mathcal{A C}$ is an ideal for $\preccurlyeq_{i}\left(\right.$ resp. $\left.\preccurlyeq_{s}\right)$ if:

1. $\forall m, t \in \mathbb{N}, z \in \mathbb{Z}: \Phi \in \mathcal{I} \Longleftrightarrow \Phi^{\langle m, t, z\rangle} \in \mathcal{I}$;

2. $\Phi_{2} \in \mathcal{I} \wedge \Phi_{1} \sqsubseteq \Phi_{2}\left(\right.$ resp. $\left.\Phi_{1} \unlhd \Phi_{2}\right) \Longrightarrow \Phi_{1} \in \mathcal{I}$;

3. $\Phi_{1} \in \mathcal{I} \wedge \Phi_{2} \in \mathcal{I} \Longrightarrow \Phi_{1} \times \Phi_{2} \in \mathcal{I}$.

Finally, let us notice that the ideal of reversible CAs is principal, as shown in [DMOT11].

\subsection{Communication complexity}

Communication complexity is a computational model designed by A. C.-C. Yao in [Yao79] to study parallel programs. In this framework, we consider two players, Alice and Bob, each with an arbitrarily high computational power, communicating to compute the value of some function $f: X \times Y \rightarrow Z$. We say that $f$ has communication complexity $c$ if, in the best protocol we can design to compute $f$ on all possible inputs $(x, y) \in X \times Y$, where Alice only knows $x$, and Bob only knows $y$, they communicate at most $c$ bits to decide the value of $f(x, y)$.

A more detailed introduction to this framework may be found in [KN97]. Here we just sum up the results and definitions important for our study. First we define what a protocol is. 
Definition 8 A protocol $\mathcal{P}$ over a domain $X \times Y$ with range $Z$ is a binary tree where each internal node $v$ is labeled either by a map $a_{v}: X \rightarrow\{0,1\}$ or by a map $b_{v}: Y \rightarrow\{0,1\}$, and each leaf $v$ is labeled either by a map $A_{v}: X \rightarrow Z$ or by a map $B_{v}: Y \rightarrow Z$.

The internal nodes of the protocol tree model communications. If a node $v$ is labeled with an $a_{v}$, Alice says one bit according to her input. If a node $v$ is labeled with an $b_{v}$, Bob says one bit according to his input. If this bit is 0 , they go on to the left child of node $v$. If it is 1 , they go on to its right child. Not surprisingly, the value of protocols, or the functions they compute, is the label of the leaf Alice and Bob arrive to if they follow all the internal nodes of the protocol tree. Hence the following definition.

Definition 9 The value of protocol $\mathcal{P}$ on input $(x, y) \in X \times Y$ is given by $A_{v}(x)$ (or $B_{v}(y)$ ) where $A_{v}$ (or $\left.B_{v}\right)$ is the label of the leaf reached by the path over the tree which starts at the root, turns left if $a_{v}(x)=0$ ( or $\left.b_{v}(y)=0\right)$, and turns right otherwise. We say that a protocol computes a function $f: X \times Y \rightarrow Z$ if for any $(x, y) \in X \times Y$, its value on input $(x, y)$ is $f(x, y)$.

We denote by $D(f)$ the (deterministic) communication complexity of a function $f: X \times Y \rightarrow Z$. It is the minimal cost of a protocol, over all protocols computing $f$, where the cost of a protocol is the depth of its corresponding tree.

In order to prove lower bounds on our constructions, we are going to use the following classical bounds on communication complexity (the proofs appear in [KN97]).

Proposition 2 Let $n \geq 1$ be fixed. Let $\mathrm{EQ}$ and DISJ be the functions "equality" and "disjointness" defined from $\{0,1\}^{n} \times\{0,1\}^{n}$ to $\{0,1\}$ by:

$$
\begin{aligned}
\mathrm{EQ}(x, y) & = \begin{cases}1 & \text { if }(\forall i)\left(x_{i}=y_{i}\right), \\
0 & \text { otherwise. }\end{cases} \\
\operatorname{DISJ}(x, y) & = \begin{cases}1 & \text { if }(\forall i)\left(x_{i} y_{i} \neq 1\right), \\
0 & \text { otherwise. }\end{cases}
\end{aligned}
$$

Both problems have maximal communication complexity, i.e. $D(\mathrm{EQ}) \geq n$ and $D(\mathrm{DISJ}) \geq n$.

In [GMRT09], there is an explanation on how to turn computational problems into communicational ones. Here we just recall the corresponding definition.

Definition 10 Let $P: Q^{+} \rightarrow Z$ be a computational problem. The communication complexity of $P$, denoted $\mathrm{CC}(P)$, is the function:

$$
n \mapsto \max _{1 \leq i \leq n-1} D\left(\left.P\right|_{n} ^{i}\right)
$$

\section{Communication complexity and simulations}

In this section, we continue the work begun in [GMRT09], incorporating two new communication problems to the three "canonical problems" developed there, and we try to extend the compatibility of these problems to the $\preccurlyeq_{s}$ relation.

In order to do this, we consider the following relation between functions from $\mathbb{R}_{+}$to $\mathbb{R}_{+}$:

$$
f_{1} \prec f_{2} \Longleftrightarrow \exists \alpha, \beta, \gamma \text { increasing affine functions, } f_{1} \circ \alpha \leq \beta \circ f_{2} \circ \gamma \text {. }
$$


Also, we use the same notation than in [GMRT09] to represent periodic configurations: if $u=u_{1} \ldots u_{l}$ is a finite word we call $p_{u}$ the infinite configuration where for all $i \in \mathbb{Z},\left(p_{u}\right)_{i}=u_{i \bmod l}$. Overmore, we denote $p_{u}\left(x_{1}, \ldots, x_{n}\right)$ the configuration obtained by modifying $p_{u}$ as follows:

$$
\left(p_{u}\left(x_{1}, \ldots, x_{n}\right)\right)_{i}= \begin{cases}\left(p_{u}\right)_{i} & \text { for } i \leq 0 \text { or } i \geq n+1, \\ x_{i} & \text { otherwise. }\end{cases}
$$

The problem called INVASION in [GMRT09], has a good behavior with respect to $\preccurlyeq_{i}$. Here, we choose to rename it in order to avoid confusions.

Definition 11 (Spatial invasion (SINv) [GMRT09]) Let $\Phi$ be a cellular automaton, and u a finite configuration for $\Phi$. The problem $\operatorname{SINV}_{\Phi}^{u}$ consists in determining whether the differences between $p_{u}$ and $p_{u}(x)$ will expand to an infinite width as times tends to infinity when applying $\Phi$ (the answer 1 means yes and the answer 0 means no).

Proposition 3 Let $\Phi$ and $\Psi$ be two cellular automata. If $\Phi \preccurlyeq_{i} \Psi$, then for all $u \in Q_{\Phi}^{+}$there exists $v \in Q_{\Psi}^{+}$ (the corresponding word by $\preccurlyeq_{i}$ ), such that:

$$
\mathrm{CC}\left(\operatorname{SINV}_{\Phi}^{u}\right) \prec \operatorname{CC}\left(\operatorname{SINV}_{\Psi}^{v}\right) .
$$

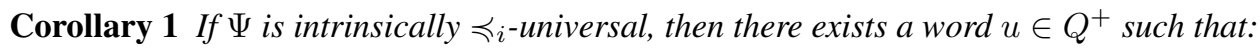

$$
\mathrm{CC}\left(\operatorname{SINV}_{\Psi}^{u}\right) \in \Omega(n) .
$$

\subsection{Temporal invasion}

Definition 12 (Temporal invasion (TINV) [GMRT09]) Let $\Phi$ be a cellular automaton, and u a finite configuration for $\Phi$. The TINV problem is the following:

$$
\operatorname{TINV}_{\Phi}^{u}(x)=\forall t,\left[t \in \mathbb{N} \Rightarrow \Phi^{t}\left(p_{u}(x)\right) \neq \Phi^{t}\left(p_{u}\right)\right] .
$$

Proposition 4 Let $\Phi$ and $\Psi$ be two cellular automata. If $\Phi \preccurlyeq_{i} \Psi$, then for all $u \in Q_{\Phi}^{+}$there exists $v \in Q_{\Psi}^{+}$ (the corresponding word by $\preccurlyeq_{i}$ ), such that:

$$
\mathrm{CC}\left(\operatorname{TINV}_{\Phi}^{u}\right) \prec \mathrm{CC}\left(\operatorname{TINV}_{\Psi}^{v}\right) \text {. }
$$

Proof: As in the other cases, we need to decompose the simulation relation:

- $\Phi^{\langle m, 1,0\rangle}$ : to simulate a protocol for $\Phi$ with a protocol for $\Phi^{\langle m, 1,0\rangle}$, Alice and Bob need to communicate $O(m)$ bits to describe the cell shared between them. The other direction is easy.

- $\Phi^{\langle 1, t, 0\rangle}$ : the protocol is exactly the same, because of the " $\forall t$ " in the definition of TINV.

- $\Phi^{\langle 1,1, z\rangle}$ : this is still the same protocol than for $\Phi$, since the worst case in the partition of the input will be the same.

- $\Phi \sqsubseteq \Psi$ : here Alice and Bob both know the injection given in the simulation. Then, they can apply it and use a protocol for $\Psi$ to solve TINV on a configuration of $\Phi$, with no overhead.

Proposition 5 There is a cellular automaton $\Phi$ and a word $u \in Q^{+}$such that $\operatorname{TINV}_{\Phi}^{u} \in \Omega(n)$. 
Proof: We reduce DISJ, a classical problem in communication complexity. We build an automaton over alphabet $Q=\{\overrightarrow{0}, \overrightarrow{1}, \overleftarrow{0}, \overleftarrow{1}, \square, \bigotimes, u\}$ with the following transition table (read it from left to right, using the first rule that applies):

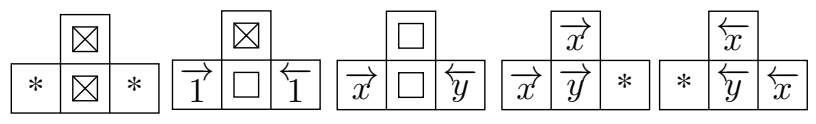

Now let $(x, y)$ an instance of DISJ, i.e. two sets of $\{1, \ldots, n\}$. An easy recursion on $n$ shows that $\operatorname{DISJ}(x, y) \Leftrightarrow \neg\left(\operatorname{TINV}_{\Phi}^{u}(\rho(x, y))\right)$, where $\rho(x, y)$ is the following configuration:

$$
\rho(x, y)=\overrightarrow{x_{n}} \ldots \overrightarrow{x_{0}} \square \overleftarrow{y_{0}} \ldots \overleftarrow{y_{n}}
$$

The recurrence hypothesis is: $\bigotimes$ appears in the orbit of $p_{u}(\rho(x, y))$ if and only if $x \cap y \neq \emptyset$ - remark that if $\square$ does not appear in any configuration of the orbit of $p_{u}(\rho(x, y))$, then all cells are in state $u$ after a finite number of steps.

Corollary 2 If $\Psi$ is intrinsically $\preccurlyeq_{i}$-universal, then there exists a word $u \in Q^{+}$such that:

$$
\operatorname{CC}\left(\operatorname{TINV}_{\Psi}^{u}\right) \in \Omega(n)
$$

\subsection{Controlled invasion and incomparability}

We shall see now a surprising connection between a well known open problem in communication complexity (the direct sum conjecture, see [KN97]), and the idea of "orthogonality" between the communication problems on cellular automata, introduced in [GMRT09].

Definition 13 (Controlled invasion (CINV)) Let $\Phi$ be a cellular automaton, and u a finite configuration for $\Phi$. The problem $\mathrm{CINV}_{\Phi}^{u}$ is defined as follows:

$$
\operatorname{CINV}_{\Phi}^{u}(x)=\operatorname{TINV}_{\Phi}^{u}(x) \wedge \neg \operatorname{SINV}_{\Phi}^{u}(x)
$$

Therefore, the output of $\mathrm{CINV}_{\Phi}^{u}(x)$ consists in determining whether the differences between $p_{u}$ and $p_{u}(x)$ persists forever but remain bounded to a finite width $1 \leq w<\infty$ when applying $\Phi$ (the answer 1 means yes and the answer 0 means no).

We shall now prove a partial result of "orthogonality" (incomparability), in the sense used in [GMRT09]: for each of the three problems SINV, TINV and CINV, we may find an automaton where it is easy, but the other ones are hard.

Proposition 6 None of the three problems SINV, TINV and CINV is stronger than the other ones.

Proof:

- Let $\Phi$ be an automaton and $u \in Q^{+}$such that $\operatorname{TINV}_{\Phi}^{u} \in \Omega(n)$, and $\operatorname{SINV}_{\Phi}^{u} \in o(n)$. Then $\Phi$ must satisfy that $\operatorname{CINV}_{\Phi}^{u} \in \Omega(n)$. If not, knowing $\operatorname{SINV}_{\Phi}^{u}$ and $\operatorname{CINV}_{\Phi}^{u}$, we could deduce $\operatorname{TINV}_{\Phi}^{u}$ with less than $\Omega(n)$ bits (indeed, $\operatorname{TINV}_{\Phi}^{u}=\operatorname{CINV}_{\Phi}^{u} \vee \operatorname{SINV}_{\Phi}^{u}$ ).

- The same proof can be used to find an automaton $\Phi$ such that $\operatorname{TINV}_{\Phi}^{u} \in o(n)$ and $\operatorname{CINV}_{\Phi}^{u} \in \Omega(n)$, for all $u \in Q^{+}$. 
- We describe here an automaton hard (i.e. with communication complexity in $\Omega(n)$ ) for TINV, SInv, but easy (in $O(1)$ ) for CINV. The idea is simple: we transform the construction of Proposition 5 by converting the $\bigotimes$ state into a spreading state. Also, we ensure the simplicity of the problem by making a $\bigotimes$ state appear each time the configuration is incorrect (i.e. not of the form $\rho(x, y)$ for some $x$ and $y$ ). This way, we get:

$$
\begin{aligned}
\operatorname{DiSJ}(x, y) & \Leftrightarrow \neg \operatorname{TINV}_{\Phi}^{u}(\rho(x, y)) \\
\operatorname{DISJ}(x, y) & \Leftrightarrow \neg \operatorname{SINV}_{\Phi}^{u}(\rho(x, y)) \\
\operatorname{CINV}_{\Phi}^{u}(\rho(x, y)) & =\operatorname{TINV}_{\Phi}^{u}(\rho(x, y)) \wedge \neg \operatorname{SINV}_{\Phi}^{u}(\rho(x, y)) \\
& =\perp
\end{aligned}
$$

Therefore, it follows that $\mathrm{CC}\left(\mathrm{CINV}_{\Phi}^{u}\right) \in O(1)$ in configurations of the form $p_{u}(\rho(x, y))$. On the other hand, in all the other configurations, a spreading state is generated and the configuration is always spatially invaded, thus $\mathrm{CC}\left(\mathrm{CINV}_{\Phi}^{u}\right) \in O(1)$.

\subsection{Non determinism and limit sets}

In order to prove non-universalities for $\preccurlyeq_{s}$ simulation, we can use the same techniques that we used previously. For instance, it is relatively simple to see why the problem PRED (see [GMRT09]) will still work in this relation. However, problems that had slightly more subtle formulations, such as TINV, formulated as "does something change?", behave in an interesting way. First, it is necessary to design a cellular automaton that we'll use in several proofs below.

\subsubsection{A convenient $C A$}

Let $\Phi_{2.3 .1}$ be a cellular automaton, product of three layers:

- The first layer operates on alphabet $Q_{1}=\{\overrightarrow{0}, \overrightarrow{1}, \overleftarrow{0}, \overleftarrow{1}, \top, \perp, S\}$. The $\vec{x}$ signals move to the right, the $\overleftarrow{x}$ signals to the left. The $T$ states change to $\perp$ whenever the symbols on its left and right are two 1s. The $\perp$ state never changes.

In any other case, transitions result in the spreading state $S$.

- The second layer operates on alphabet $Q_{2}=\{\leftarrow, \leftarrow, \bullet\}$.

Whenever —on the first layer — the $T$ state has two 1 s signals on its sides, $\mathrm{a} \leftarrow$ is generated, moving to the left.

When the signals' contents are other than two $1 \mathrm{~s}, \mathrm{a} \leftarrow$ appears, also moving to the left.

Also, $\bullet$ is a quiescent state.

- The third layer is like the second one, but on alphabet $Q_{3}=\{\rightarrow, \neg, \bullet\}$, moving to the right.

We shall argue now that this automaton has a trivial SINV problem, as well as a trivial TINV problem. Indeed, there are three cases for the background $u$ :

1. If it has a $\vec{x}$ or $\overleftarrow{x}$ signal on the first layer, then all the other states must be signals in the same direction. In this case, the configuration is invaded if and only if the input is anything else than signals in this direction: a spreading state is generated on this component. 
2. If it has a spreading state, no invasion can occur.

3. Otherwise, the background can be only $\diamond$, in which case invasion occurs, possibly on the second layer, if and only if the input is not only signals in the same direction.

In all three cases, the property can be checked by Alice and Bob with very few communicated bits, thus answering to the SINV problem. Since the configuration is changed, the TINV problem is also easy.

Proposition 7 There is a cellular automaton $\Psi$ such that $\Psi \unlhd \Phi_{[2.3 .1}$ and:

$$
\mathrm{CC}\left(\operatorname{SINV}_{\Psi}\right) \in \Omega(n) .
$$

Proof: If the quotient relation identifies states $\angle$ and $\diamond$ on the second component, $\rightarrow$ and $\bullet$ on the third component, there is a set of configurations for which the problem becomes as difficult as the DISJ problem, defined in [KN97], i.e. $\mathrm{CC}\left(\operatorname{SINV}_{\Psi}\right) \in \Omega(n)$.

Proposition 8 There is a cellular automaton $\Psi$ such that $\Psi \unlhd \Phi_{2.3 .1}$ and:

$$
\mathrm{CC}\left(\operatorname{TINV}_{\Psi}\right) \in \Omega(n) .
$$

Proof: If the quotient relation identifies all states with $\bullet$ on the second component and all states with $\checkmark$ on the third component, on configurations of the form $\vec{*}^{n} \top^{\leftarrow *}$, solving the problem TINV requires deciding if the middle $T$ symbol turns $\perp$ somewhere in the space-time diagram, which is as difficult as the DISJ problem, defined in [KN97], i.e. $\mathrm{CC}\left(\operatorname{TINV}_{\Psi}\right) \in \Omega(n)$.

This raises new questions: is this simulation stronger or weaker than the previous one? As studied in [DMOT11], we know that they are incomparable. In this section, we introduce a problem whose communication complexity grows with respect to $\preccurlyeq_{s}$, but for which it is not the case in relation $\preccurlyeq_{i}$. At the same time, it may be a clue that our approach with communication complexity will not be able to tell much about Open Problem 1

Definition 14 (Limit set word (LIMIT)) LIMIT $\Phi$ is the problem of deciding if the input word belongs to the language of the limit set of $\Phi$ :

$$
\begin{aligned}
& \operatorname{Limit}_{\Phi}(x)= \begin{cases}1 & \text { if } \forall t, \exists y, x=\Phi^{t}(y), \\
0 & \text { otherwise. }\end{cases} \\
& \text { Let } \\
& \mathrm{CC}\left(\operatorname{Limit}_{\Phi}\right)=\max _{i} D\left(\operatorname{LimiT}_{\Phi}\left(x_{[0, i]}, x_{[i+1, n-1]}\right)\right)
\end{aligned}
$$

be the deterministic communication complexity of this problem, and

the non-deterministic version.

$$
\operatorname{NCC}\left(\operatorname{LiMit}_{\Phi}\right)=\max _{i} N^{1}\left(\operatorname{Limit}_{\Phi}\left(x_{[0, i]}, x_{[i+1, n-1]}\right)\right)
$$

Now we need to show that the non-deterministic communication complexity of this problem grows with respect to the $\preccurlyeq s$ relation. As in the other definitions (see [GMRT09], and Subsection 2.1, we only need to show that the complexity is preserved with each ingredient of the simulation: 


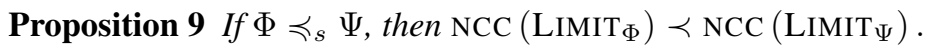

Proof: We showed that rescaling did not change the communication complexity of similar problems in the deterministic case; we can use exactly the same proof here, in the non-deterministic case.

For the quotient relation, if $\varphi: Q_{\Psi}^{m_{\Psi}} \rightarrow Q_{\Phi}^{m_{\Phi}}$ is the quotient map, and $\bar{\varphi}$ is its uniform extension to infinite configurations, if at least one element $x$ of $(\bar{\varphi})^{-1}(x)$ is in $\omega(\Psi)$, then $\bar{\varphi}(x) \in \omega(\Phi)$. Then, we use the non-determinism of the protocol to choose the correct traslation of each of the cells: at the first step of the protocol where Alice (resp. Bob) speaks, they take a non-deterministic step to choose a traslation of their configuration that leads to a positive answer if there is any. We insist on the fact that this step needs to non-deterministically choose a traslation and keep it for the rest of the protocol.

Proposition 10 There exists a cellular automaton $\Phi$ such that:

$$
\operatorname{NCC}\left(\operatorname{Limit}_{\Phi}\right) \in \Omega(n) .
$$

Proof: We consider a cartesian product of three layers:

1. A shift to the left, i.e. $\sigma$ over alphabet $\{0,1\}$.

2. A shift to the right, i.e. $\sigma^{-1}$ over alphabet $\{0,1\}$.

3. A test layer with three states: a blank state, a "test" state, and a "corrupt test" state. This is a cellular automaton of radius 0 , with the following rule:

- The blank state remains blank.

- The corrupt test remains corrupt.

- Whenever a test sees a 1 on both of the two other layers, it gets corrupt. Otherwise, it remains a normal test.

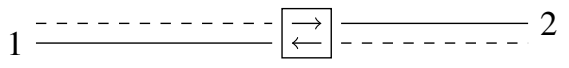

Fig. 1: An automaton hard for LiMIT.

We only need to find a set of configurations big enough, and hard for the LiMIT problem: the configurations of odd size, with one test cell in the middle of the third layer, are hard. Indeed, for this configuration to be in the limit set, an infinite DISJ problem needs to be solved between the word on layer 1, and the mirror of the word on layer 2 (i.e. on the whole lines on Figure 11, so Alice and Bob need to solve an instance of DISJ in order to find out whether the input belongs to the limit set, which, according to [KN97], requires $\Omega(n)$ bits to be solved by a non-deterministic protocol.

This shows that this problem is compatible with the $\preccurlyeq s$ relation, i.e. that the following holds.

Corollary 3 If $\Psi$ is intrinsically $\preccurlyeq{ }_{s}$-universal, then:

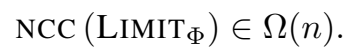

Now, according to the analysis of [GMT10], there exists a cellular automaton universal for $\preccurlyeq_{i}$, with complexity not greater than $O(\log n)$ for the LIMIT problem.

This problem may seem an odd counter-example. However, we will see in Proposition 14 how to use it to show that no stable automaton can be intrinsically $\preccurlyeq$-universal. 


\section{Structuring communication problems}

In this section, we explore the links between our approach using communication complexity to study cellular automata and the general theory of bulking, developed in [DMOT10, DMOT11]. In order to do this, we prove the existence of new ideals and inclusions, giving a more detailed vision of quasi-orders induced by simulations. As showing that a given CA belongs to an ideal is a way to prove that it cannot be intrinsically universal, we illustrate here how our tools adapt well to this framework and extend it, being the communication approach, to our knowledge, the best way of proving non-universality in cellular automata.

\subsection{Closing CAs}

In the following, we ennunciate some results concerning the sets of closing and open CAs. As we suspect, this is closely related with the following open problem.

Open Problem 2 ([DMOT11]) Is the ideal of surjective CA principal, and for which simulation quasiorder?

Definition 15 (Asymptotic configurations [K0ㅇ]) $x, y \in Q^{\mathbb{Z}}$ are left (right) asymptotic, if there exists $m \in \mathbb{Z}$ such that $x_{i}=y_{i}$ for all $i \leq m$ (for all $i \geq m$ ).

Definition 16 (Closingness [K0ㅇ]) A CA $\Phi$ is right closing (resp. left closing), if for every distinct left asymptotic (right asymptotic) $x, y \in Q^{\mathbb{Z}}, \Phi(x) \neq \Phi(y)$. A CA is closing if it is either left or right closing. Clearly,

$$
\Phi \text { injective } \Longrightarrow \Phi \text { closing } \Longrightarrow \Phi \text { preinjective } \Longleftrightarrow \Phi \text { surjective. }
$$

Theorem 1 Let $\preccurlyeq \in\{\preccurlyeq i, \preccurlyeq s\}$. Then, the set of right closing (left closing) CAs is an ideal for $\preccurlyeq$.

Proof: See the Appendix.

Proposition 11 ([广]еd69]) A CA is open if and only if is right-closing and left-closing.

Corollary 4 Let $\preccurlyeq \in\{\preccurlyeq i, \preccurlyeq s\}$. Then, the set of open CAs is an ideal for $\preccurlyeq$.

\subsection{Stable CAs}

As proved in [GMT10], the set of stable cellular automata is an ideal for $\preccurlyeq s$. Nevertheless, its behavior with respect to relation $\preccurlyeq_{i}$ is unclear. We do not even know whether there is any stable universal automaton. However, the following results might help.

Lemma 1 Let $\Phi \in \mathcal{A C}$. Then, if $\omega(\Phi)$ is an SFT, $\Phi$ is stable.

Proof: A SFT subshift is characterized by a finite set of forbidden words $F$. For each $w \in F$, by compactness, there is a first time step $t_{w}$ in which word $w$ does not appear anymore in $\Phi^{t_{w}}\left(Q^{\mathbb{Z}}\right)$. Taking $t^{*}=\max _{w \in F} t_{w}$, it follows that $\omega(\Phi)=\Phi^{t^{*}}\left(Q^{\mathbb{Z}}\right)$.

Lemma 2 Let $\Phi \in \mathcal{A C}$. Then, if $\omega(\Phi)$ is an SFT, $\Phi$ is preinjective restricted to its limit set.

Proof: The result follows directly by considering the more general case of an onto sliding block code from an irreducible SFT (for a further explanation, see [LM95]). 
Proposition 12 Let $\Phi$ be a CA with SFT limit set. Then, for all $u \in Q^{+}$:

$$
\operatorname{CC}\left(\operatorname{TINV}_{\Phi}^{u}\right) \in O(1)
$$

Proof: By Lemma 1, all CA with SFT limit set are stable. Therefore, there exists a time $t^{*}$ such that $\omega(\Phi)=\Phi^{t^{*}}\left(Q^{\mathbb{Z}}\right)$. Then, the protocol just consists of iterating $t^{*}$ times the periodic configuration and the perturbation (this has constant cost) and reach the limit set. Later, Alice and Bob only have to check if they have some difference with respect to the non perturbated pattern (which also has constant cost). As $\Phi$ is preinjective on $\omega(\Phi)$ (by Lemma 2), finite differences will remain forever. Therefore, the whole protocol has constant cost and stable intrinsically universal CAs cannot exist.

Corollary 5 No cellular automaton with a SFT limit set can be intrinsically $\preccurlyeq_{i}$-universal.

Proposition 13 Let $\Phi$ be a $C A$ with sofic limit set. Then:

$$
\mathrm{CC}\left(\operatorname{Limit}_{\Phi}\right) \in O(1) \text {. }
$$

Proof: The language of the limit set $\omega(\Phi)$ of such a CA is regular, recognized by a finite automaton $\mathcal{A}$. Knowing both Alice and Bob $\mathcal{A}$, the only thing Alice needs to say to solve $\operatorname{Limit}_{\Phi}$ is the state she gets on it after having read her half of the configuration, if possible, and answer 0 elsewhere.

A result that follows directly from Proposition 13 is that no stable CA can be $\preccurlyeq s$-universal, because every stable CA has a sofic limit set. However, they accept a different protocol with constant cost, besides the previous one for the more general case.

Proposition 14 Let $\Phi$ be a stable CA. Then:

$$
\mathrm{CC}\left(\operatorname{LiMIT}_{\Phi}\right) \in O(1) .
$$

Proof: If $\omega(\Phi)=\Phi^{t^{*}}\left(Q^{\mathbb{Z}}\right)$ and $(\phi, r)$ is the canonical local representation of $\Phi$, Alice only has to send to Bob the $2\left(t^{*}+1\right) r$ rightmost bits of each of the possible antecedents of her input. There may be many of them, but there are just $|Q|^{2\left(t^{*}+1\right) r}$ combinations of the relevant parts of the configuration (which has constant cost for our purposes). Later, Bob can verify by his own if the input belongs to $\omega(\Phi)$ or don't.

Corollary 6 No cellular automaton with a sofic limit set can be intrinsically $\preccurlyeq{ }_{s}$-universal. In particular, no stable CA can be intrinsically $\preccurlyeq{ }_{s}$-universal.

\subsection{Communication ideals}

The last proposition showed another example of a suprising correlation between ideals and simple protocols, that seems to generalize in a way that we were not completely able to formalize until now. Although the notion of uniformity among protocols, which we need here, seems difficult to formalize, probably due to the generality of the communication approach, the following proposition may be a first step in this direction: 


\begin{tabular}{lcc}
\hline Ideals & $\preccurlyeq_{i}$ & $\preccurlyeq_{s}$ \\
\hline Sofic limit set & $?$ & $\checkmark$ \\
Stable limit set & $?$ & \\
SFT limit set & $?$ & \\
Surjective & $\checkmark$ & \\
Closing & $\checkmark$ & $\checkmark$ \\
Open & $\checkmark$ & $\checkmark$ \\
Injective & $\checkmark$ & $\checkmark$ \\
Positive expansive & $\checkmark$ & $?$ \\
Nilpotent over periodic configurations & & \\
\hline
\end{tabular}

Tab. 1: Relevant known ideals.

Proposition 15 Let $\left(X_{\Phi}\right)_{\Phi \in \mathcal{A C}}$ a family of communication problems, defined for each cellular automaton, of complexity increasing with respect to simulation $\preccurlyeq\left(\right.$ i.e. if $\Phi \preccurlyeq \Psi$, then $\left.\mathrm{CC}\left(X_{\Phi}\right) \prec \operatorname{CC}\left(X_{\Psi}\right)\right)$. Let $f$ be a non-decreasing function from $\mathbb{N} \rightarrow \mathbb{N}$. Then the following set is an ideal for $\preccurlyeq$ :

$$
\mathcal{I}=\left\{\Phi \in \mathcal{A C} \mid \mathrm{CC}\left(X_{\Phi}\right) \prec f\right\} .
$$

Now, what can be these new ideals? As the following example shows, their "shape" is quite undefined and might be complicated. Indeed, we showed in proposition 14 that stable CA had a simple protocol for Limit. Now we show an example of the same class of communication complexity, this time unstable:

Example 1 Is easy to show that there exists an unstable $C A \Phi$ such that, for all $u \in Q^{+}, \operatorname{CC}_{\left(\operatorname{LIMIT}_{\Phi}\right) \in}$ $O(1)$. In fact, to see this, consider the "multiplication" automaton, on alphabet $\{0,1\}$, given by the local rule:

$$
\phi\left(x_{i-1}, x_{i}, x_{i+1}\right)=x_{i-1} \cdot x_{i} \cdot x_{i+1}
$$

This CA is unstable, since for all integer $t$, configurations of the form ${ }^{\infty} 010^{2 t} 010^{\infty}$ have an antecedent by $\Phi^{t}$ but not by $\Phi^{t+1}$. Now, it can be checked that for all $u \in\{0,1\}^{+}: \mathrm{CC}\left(\operatorname{TINV}_{\Phi}^{u}\right) \in O(1)$. To see this, notice that a configuration $x \in\{0,1\}^{\mathbb{Z}}$ converges to ${ }^{\infty} 0^{\infty}$ if and only if $x \neq \neq^{\infty} 1^{\infty}$. Then, a protocol for LIMIT $_{\Phi}^{u}$ only has to check that Alice and Bob have only 0 s ore only 1 s.

This shows that communication complexity may allow us to describe a large number of complicated ideals in a really simple way. We are now just missing a finer definition of "class of protocols"...

\section{Conclusion and perspectives}

The theory of bulking and intrinsic universality in cellular automata is a fascinating topic, and communication complexity seems well suited to study this complexity.

Among the many open problems and perspectives, we would like to emphasize the following ones:

- How can we characterize a stable family of protocols? Each communication problem we studied until now was proved increasing by simulation in a way pretty similar to caracterizations of ideals. What are the exact relations, and how can simple protocols give us ideals "for free"? 
- Although [GMT10] proved that there are few relations between the complexity of the limit set and intrinsic universality, it seems impossible that the limit sets of $\preccurlyeq$-instrinsic universal CAs be as simple as envisioned in that paper. An automaton with a sofic limit set can have sub-automata with more complex limit sets? What about SFT limit sets?

- Here, and for the first time, we were forced to introduce non-determinism in our proofs of compatibility between simulation and communication complexity. What does this tell us on the relation between the two simulations?

- Considering Example 1, how complex can be the limit set of an unstable CA $\Phi$ such that, for all $u \in Q^{+}, \operatorname{CC}\left(\operatorname{TINV}_{\Phi}^{u}\right) \in O(1)$ ?

- Until now, we only have used deterministic protocols to prove non-universality. Simulation $\preccurlyeq s$ did not give us the choice: it seems that we really need non-determinism in the proof of proposition 9 Why does this happen? With what consequences?

- In $[\overline{B R}]$ is showed a way to generalize the framework of communication complexity, using relations instead of functions and giving an unification of some of the problems developed here and in [GMRT09]. What happen when non-determinism is used as in LIMIT? Is there any way to incorporate it to that technique?

\section{Acknowledgement}

We would like to thank Mike Boyle for a helpful discussion about closing CAs, as well as Guillaume Theyssier for the proof of Lemma 1

\section{References}

[BR] R. Briceño and I. Rapaport. Letting Alice and Bob choose which problem to solve: implications to the study of cellular automata (prepublication).

[BT10] L. Boyer and G. Theyssier. On factor universality in symbolic spaces. In P. Hlinený and A. Kucera, editors, MFCS, volume 6281 of Lecture Notes in Computer Science, pages 209-220. Springer, 2010.

[DMOT10] M. Delorme, J. Mazoyer, N. Ollinger, and G. Theyssier. Bulking I: an abstract theory of bulking. HAL:hal-00451732, 2010.

[DMOT11] M. Delorme, J. Mazoyer, N. Ollinger, and G. Theyssier. Bulking II: Classifications of cellular automata. Theor. Comput. Sci., 412(30):3881-3905, 2011.

[GMRT09] E. Goles, P.-E. Meunier, I. Rapaport, and G. Theyssier. Communication complexity and intrinsic universality in cellular automata. CoRR, abs/0912.1777, 2009.

[GMT10] P. Guillon, P.-E. Meunier, and G. Theyssier. Clandestine simulations in cellular automata. CoRR, abs/1009.5621, 2010.

[Hed69] G. A. Hedlund. Endomorphisms and automorphisms of the shift dynamical systems. Mathematical Systems Theory, 3(4):320-375, 1969.

[Jun09] U. Jung. On the existence of open and bi-continuing codes. arXiv:0810.4632v2, 2009.

[KN97] E. Kushilevitz and N. Nisan. Communication complexity. Cambridge university press, 1997. 
[K09] P. Kůrka. Topological dynamics of cellular automata. In Encyclopedia of Complexity and Systems Science, pages 9246-9268. Springer, 2009.

[LM95] D. Lind and B. Marcus. An introduction to symbolic dynamics and coding. Cambridge University Press, 1995.

[O1103] N. Ollinger. The intrinsic universality problem of one-dimensional cellular automata. In STACS, pages 632-641, 2003.

[The05] G. Theyssier. Cellular automata : a model of complexities. PhD thesis, ENS Lyon, 2005.

[Yao79] A. C.-C. Yao. Some complexity questions related to distributive computing (preliminary report). In STOC, pages 209-213. ACM, 1979.

\section{Appendix A: Proof, ideal of closing CAs}

Proof: In order to prove this, we adopt an enumeration like in Proposition 1

(1) Let $m, t \in \mathbb{N}, z \in \mathbb{Z}$. Then:

- $\Phi$ es right closing $\Longleftrightarrow b_{m} \circ \Phi \circ b_{m}^{-1}$ is right closing.

In fact, if $\Phi$ is right closing, suppose there exist different left asymptotic configurations $x, y \in\left(Q^{m}\right)^{\mathbb{Z}}$ such that: $\Phi^{\langle m, 1,0\rangle}(x)=\Phi^{\langle m, 1,0\rangle}(y)$. Then,

$$
b_{m} \circ \Phi \circ b_{m}^{-1}(x)=b_{m} \circ \Phi \circ b_{m}^{-1}(y) .
$$

As $\gamma_{m}$ is bijective, it follows that:

$$
\Phi \circ b_{m}^{-1}(x)=\Phi \circ b_{m}^{-1}(y)
$$

Therefore, $b_{m}^{-1}(x), b_{m}^{-1}(y) \in Q^{\mathbb{Z}}$ are different left asymptotic configurations and their images via $\Phi$ are equal, which is a contradiction.

On the other hand, suppose that $\Phi^{\langle m, 1,0\rangle}$ is right closing and there exist different left asymptotic configurations $x, y \in Q^{\mathbb{Z}}, x \neq y$ such that:

$$
\begin{aligned}
& \Phi(x)=\Phi(y) \\
& \Longrightarrow \quad \Phi \circ b_{m}^{-1}\left(b_{m}(x)\right)=\Phi \circ b_{m}^{-1}\left(b_{m}(y)\right) \\
& \Longrightarrow \quad b_{m} \circ \Phi \circ b_{m}^{-1}\left(b_{m}(x)\right)=b_{m} \circ \Phi \circ b_{m}^{-1}\left(b_{m}(y)\right) \\
& \Longrightarrow \quad \Phi^{\langle m, 1,0\rangle}\left(b_{m}(x)\right)=\Phi^{\langle m, 1,0\rangle}\left(b_{m}(y)\right),
\end{aligned}
$$

but this is a contradiction, because $b_{m}(x), b_{m}(y) \in\left(Q^{m}\right)^{\mathbb{Z}}$ are different left asymptotic configurations.

- $\Phi$ is right closing $\Longleftrightarrow \Phi^{t}$ is right closing.

Suppose that $\Phi$ is right closing. Then, if $x, y \in Q^{\mathbb{Z}}$ are different left asymptotic configurations, $\Phi(x), \Phi(y) \in$ $Q^{\mathbb{Z}}$ too. Iterating the argument, it can be concluded that $\Phi^{t}(x) \neq \Phi^{t}(y)$.

By other side, if $\Phi^{t}$ is right closing and $x, y \in Q^{\mathbb{Z}}$ are different left asymptotic configurations, it follows that $\Phi^{t}(x) \neq \Phi^{t}(y)$ and this implies that necessarily $\Phi(x) \neq \Phi(y)$.

- $\Phi$ is right closing $\Longleftrightarrow \sigma^{z} \circ \Phi$ is right closing.

Notice that $x, y \in Q^{\mathbb{Z}}$ are different left asymptotic configurations if and only if $\sigma^{z}(x)$ and $\sigma^{z}(y)$ satisfy that, too. Therefore,

$$
\begin{aligned}
& \Phi\left(\sigma^{z}(x)\right) \neq \Phi\left(\sigma^{z}(x)\right) \\
& \Longrightarrow \quad \sigma^{z} \circ \Phi(x) \neq \sigma^{z} \circ \Phi(y) \\
& \Longrightarrow \quad \Phi(x) \neq \Phi(y) .
\end{aligned}
$$

All this by the commutativity of the shift and its bijectivity. 
Then, composing all the partial results, we conclude.

(2) For the two simulation relations:

- $\sqsubseteq$ : let $\Phi_{2} \in \mathcal{A C}$ be right closing and $\Phi_{1} \in \mathcal{A C}$ such that $\Phi_{1} \sqsubseteq_{\bar{\iota}} \Phi_{2}$. Then, if $x, y \in Q_{1}^{\mathbb{Z}}$ are distinct left asymptotic configurations, then $\bar{\imath}(x), \bar{\iota}(y) \in A_{2}^{\mathbb{Z}}$ satisfy that, too. Therefore,

$$
\bar{\iota} \circ \Phi_{1}(x)=\Phi_{2} \circ \bar{\iota}(x) \neq \Phi_{2} \circ \bar{\iota}(y)=\bar{\iota} \circ \Phi_{1}(y) .
$$

Then, $\bar{\iota} \circ \Phi_{1}(x) \neq \bar{\iota} \circ \Phi_{1}(y)$, which implies that $\Phi_{1}(x) \neq \Phi_{1}(y)$.

- $\unlhd$ : the proof is in Subsection Proof: quotient of closing CAs.

(3) Let $\Phi_{1}, \Phi_{2} \in \mathcal{A C}$ be right closing. Then, their cartesian product $\Phi_{1} \times \Phi_{2}$ is also right closing. In fact, let $\left(x_{1}, x_{2}\right),\left(y_{1}, y_{2}\right) \in Q_{\Phi_{1} \times \Phi_{2}}^{\mathbb{Z}}$ be distinct left asymptotic configurations. Then, so does one of the pairs $x_{1}, y_{1}$ or $x_{2}, y_{2}$. Then, by the closingness of $\Phi_{1}$ o $\Phi_{2}$, respectively, it follows that:

$$
\Phi_{1} \times \Phi_{2}\left(x_{1}, x_{2}\right)=\left(\Phi_{1}\left(x_{1}\right), \Phi_{2}\left(x_{2}\right)\right) \neq\left(\Phi_{1}\left(y_{1}\right), \Phi_{2}\left(y_{2}\right)\right)=\Phi_{1} \times \Phi_{2}\left(x_{1}, x_{2}\right) .
$$

The proof for the left closing case is analogous.

\section{Appendix B: Proof, quotient of closing CAs}

Definition 17 (Entropy [LM95]) Let $X$ be a space shiftt. We define the entropy of $X$ as follows:

$$
h(X)=\lim _{n \rightarrow \infty} \frac{1}{n} \log \left|\mathcal{L}_{n}(X)\right|
$$

Lemma 3 (|[LM95|) Let $\Phi: X \rightarrow Y$ be a sliding block code. Then, $\Phi$ is right closing if and only if:

$$
\exists N \in \mathbb{N}: x_{[-N, 0]}=y_{[-N, 0]} \wedge \Phi(x)_{[-N, N]}=\Phi(y)_{[-N, N]} \Longrightarrow x_{1}=y_{1} .
$$

The case of a left closing CA is analogous.

Definition 18 A sliding block code $\Phi: X \rightarrow Y$ is a 1-block code if it can be induced by a 1-block map with memory $m=0$ and anticipation $a=0$, namely, it exists $\phi: \mathrm{A}(X) \rightarrow \mathrm{A}(Y)$ such that:

$$
\Phi(x)_{i}=\phi\left(x_{i}\right)
$$

for all $i \in \mathbb{Z}$, for all $x \in X$.

Definition 19 A SFT $X$ is said to be $M$-step if it can be defined by a family of forbidden words $F \subseteq A(X)^{M+1}$.

Definition 20 A sliding block code $\Phi: X \rightarrow Y$ is a conjugacy between $X$ and $Y$ if it is invertible. Two shift spaces are conjugated if there exists a conjugacy between them.

Proposition 16 Let $\Phi: X \rightarrow Y$ be a sliding block code. Then, exists a shift space $\tilde{X}$, a conjugacy $\pi: X \rightarrow \tilde{X}$ and a 1-block code $\tilde{\Phi}: \tilde{X} \rightarrow Y$ such that $\tilde{\Phi} \circ \pi=\Phi$, that is to say, the following diagram commutes:

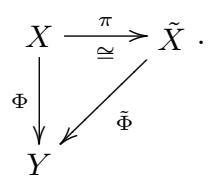


Proof: Suppose that $\Phi$ have memory $m$, anticipation $a$ and it is induced by a block map $\phi$. Let $\pi: X \rightarrow$ $\mathcal{L}_{m+a+1}(X)^{\mathbb{Z}}$ such that $\pi(x)_{[i]}=x_{[i-m, i+a]}$. Then, $\pi=\sigma^{-m} \circ \beta_{m+n+1}$. Therefore, $\tilde{X}=\pi(X)=X^{[m+n+1]}$ is a shift space and, because $\sigma$ and $\beta_{m+n+1}$ are conjugacies, $\pi$ is a conjugacy, too. Considering $\tilde{\Phi}=\Phi \circ \pi^{-1}$, the result follows. Finally, note that $\tilde{\Phi}$ is a 1-block code.

Definition 21 Let $S$ and $T$ be two SFT. A 1-block code $\Phi: S \rightarrow T$ is said to be e-right-resolving if, given $b_{1} b_{2} \in$ $\mathcal{L}(T)$ and $a_{1} \in \mathrm{A}(S)$ such that $\phi\left(a_{1}\right)=b_{1}$, there exists $a_{2} \in \mathrm{A}(S)$ such that $\phi\left(a_{2}\right)=b_{2}$ and $a_{1} a_{2} \in \mathcal{L}(S)$.

Definition 22 Let $S$ and $T$ be two SFT. A 1-block code $\Phi: S \rightarrow T$ is said to be u-right-resolving if, given $b_{1} b_{2} \in$ $\mathcal{L}(T)$ and $a_{1} \in \mathrm{A}(S)$ such that $\phi\left(a_{1}\right)=b_{1}$, there is one and only one $a_{2} \in \mathrm{A}(S)$ such that $\phi\left(a_{2}\right)=b_{2}$ and $a_{1} a_{2} \in \mathcal{L}(S)$.

Remark 1 Clearly, every u-right-resolving 1-block code is e-right-resolving, too. Nevertheless, there exist examples where the other implication is not true.

Sliding block codes which are right closing can be characterized as those which can be conjugated to an u-rightresolving 1-block code. There exists an analogous characterization between e-right-resolving 1-block codes and a family of sliding block codes called right continuing. Here we state the non trivial implication.

Proposition 17 Let $\Phi: X \rightarrow Y$ be a right closing sliding block code. Then, there exists a space shift $X^{\prime}$, a conjugacy $\Theta: X^{\prime} \rightarrow X$ and an u-right-resolving 1-block code $\Phi^{\prime}$ such that $\Phi^{\prime} \circ \Theta=\Phi$, that is to say, the following diagram commutes:

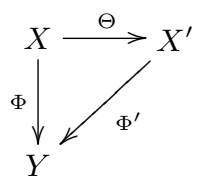

Proof: Without loss of generality, by Proposition 16 it can be considered $\Phi$ as a right closing 1-block code, because right closing property is an invariant under conjugacies. By Lemma 3 there exists $N \in \mathbb{N}$ such that:

$$
x_{[-N, 0]}=x_{[-N, 0]}^{\prime} \wedge \Phi(x)_{[-N, N]}=\Phi\left(x^{\prime}\right)_{[-N, N]} \Longrightarrow x_{1}=x_{1}^{\prime} .
$$

Given $N$, consider the equivalence relation $\bumpeq$ defined over $\mathcal{L}_{2 N+1}(X)$ as:

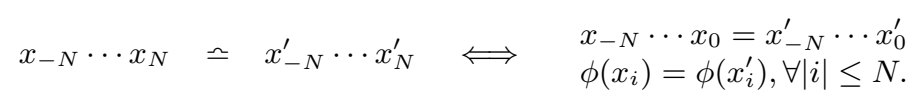

Later, define $\mathrm{A}\left(X^{\prime}\right)$ as $\mathcal{L}_{2 N+1}(X) / \bumpeq$, this is to say:

$$
\mathrm{A}\left(X^{\prime}\right)=\left\{W\left(x_{-N} \cdots x_{0} ; y_{-N} \cdots y_{N}\right): \begin{array}{lll}
x_{-N} \cdots x_{0} & \in \mathcal{L}(X) \\
y_{-N} \cdots y_{N} & \in & \mathcal{L}(Y)
\end{array}\right\},
$$

where:

$$
W\left(x_{-N} \cdots x_{0} ; y_{-N} \cdots y_{N}\right)=\left\{x_{-N}^{\prime} \cdots x_{N}^{\prime} \in \mathcal{L}_{2 N+1}(X): \begin{array}{l}
x_{-N} \cdots x_{0}=x_{-N}^{\prime} \cdots x_{0}^{\prime} \\
\phi\left(x_{i}\right)=\phi\left(x_{i}^{\prime}\right), \forall|i| \leq N .
\end{array}\right\} .
$$

Then, define $X^{\prime}$ as a 1-step subshift such that:

$$
W\left(x_{-N} \cdots x_{0} ; y_{-N} \cdots y_{N}\right) W\left(x_{-N}^{\prime} \cdots x_{0}^{\prime} ; y_{-N}^{\prime} \cdots y_{N}^{\prime}\right) \in \mathcal{L}_{2}\left(X^{\prime}\right)
$$


if and only if:

$$
x_{-N+1} \cdots x_{0}=x_{N}^{\prime} \ldots x_{1}^{\prime}, y_{-N+1} \cdots y_{N}=y_{-N}^{\prime} \cdots y_{N-1}^{\prime},
$$

being $x_{0}^{\prime}$ the state determined by $x_{-N} \cdots x_{0}$ and $y_{-N} \cdots y_{N}$, because the right closingness of $\Phi$.

Considering that, it is defined the 1-block code $\Theta: X^{\prime} \rightarrow X$ by the block map:

$$
\theta\left(W\left(x_{-N} \cdots x_{0} ; y_{-N} \cdots y_{N}\right)\right)=x_{0},
$$

with local inverse given by:

$$
\theta^{-1}\left(x_{-N}, \ldots, x_{N}\right)=W\left(x_{-N} \cdots x_{0} ; \phi\left(x_{-N}\right) \cdots \phi\left(x_{N}\right)\right) .
$$

Finally, is defined the 1-block code $\Phi^{\prime}: X^{\prime} \rightarrow Y$ by the block map:

$$
\phi^{\prime}\left(W\left(x_{-N} \cdots x_{0}, y_{-N} \cdots y_{N}\right)\right)=y_{N}
$$

this is to say, $\Phi^{\prime}=\Phi \circ \sigma^{N} \circ \Theta$. Therefore, $\Phi^{\prime}$ is u-right-resolving. In fact, suppose there exist $y_{N} y_{N+1} \in \mathcal{L}(Y)$ and $W \in \mathrm{A}\left(X^{\prime}\right)$ such that $\phi^{\prime}(W)=y_{N}$. Then, $W$ should have the following structure:

$$
W=W\left(x_{-N} \cdots x_{0} ; y_{-N} \cdots y_{N}\right) .
$$

By definition of $X^{\prime}$, any $W^{\prime} \in \mathrm{A}\left(X^{\prime}\right)$ such that $W W^{\prime} \in \mathcal{L}\left(X^{\prime}\right)$ must satisfy that:

$$
W^{\prime}=W\left(x_{-N+1} \cdots x_{0} a ; y_{-N+1} \cdots y_{N} b\right),
$$

where $a$ and $b$ must be determined. The value of $b$ is determined by $y_{N+1}$ and because $\phi^{\prime}\left(W^{\prime}\right)=y_{N+1}$. Finally, $a$ is determined (both existence and uniqueness) by the right closingness of $\Phi$. Then, the result follows.

Lemma 4 ([ذاJun09]) Let $\Phi: S \rightarrow T$ be an e-right-resolving 1-block code, with $S$ and $T$ two irreducible SFT such that $h(S)=h(T)$. Therefore, $\Phi$ is u-right-resolving.

Proposition 18 Let $\Phi: X \rightarrow X$ and $\Psi: Y \rightarrow Y$ be two CAs, such that $\Psi$ is right closing and $\Phi \unlhd \Psi$. Therefore, $\Phi$ is right closing.

Proof: Let $\varphi: A(X) \rightarrow A(Y)$ such that $\Phi \unlhd_{\varphi} \Psi$. The hypothesis of the proposition can be represente by the following diagram:

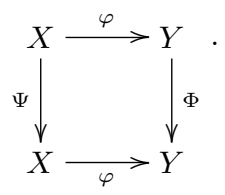

By Proposition 16 there exist shift spaces $\tilde{X}$ and $\tilde{Y}$, conjugacies $\pi_{X}$ and $\pi_{Y}$, and 1-block codes $\tilde{\Psi}: \tilde{X} \rightarrow X$ and $\tilde{\Phi}: \tilde{X} \rightarrow X$ such that $\tilde{\Psi} \circ \pi_{X}=\Psi$ and $\tilde{\Phi} \circ \pi_{X}=\Phi$, respectively. Then, we have the following completion of the previous diagram:

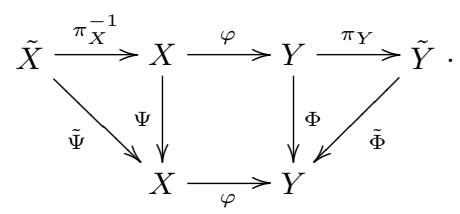


Applying the previous argument to $\pi_{X}^{-1} \circ \varphi \circ \pi_{Y}$, there exists a shift space $\bar{X}$, a conjugacy $\pi_{\tilde{X}}$ and a 1-block code $\overline{\left(\pi_{X}^{-1} \circ \varphi \circ \pi_{Y}\right)}: \bar{X} \rightarrow \tilde{Y}$ such that $\overline{\left(\pi_{X}^{-1} \circ \varphi \circ \pi_{Y}\right)} \circ \pi_{\tilde{X}}=\pi_{X}^{-1} \circ \varphi \circ \pi_{Y}$.

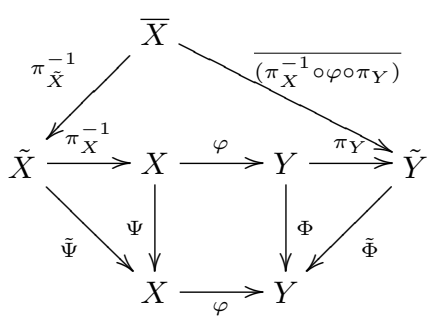

Then, renaming variables $\left(X_{1}=\bar{X}, X_{2}=X, Y_{1}=\tilde{Y}, Y_{2}=Y, \varphi_{1}=\overline{\left(\pi_{X}^{-1} \circ \varphi \circ \pi_{Y}\right)}\right.$ у $\varphi_{2}=\varphi, \Psi_{X}=$ $\left.\tilde{P s i} \circ \pi_{\tilde{X}}^{-1}\right)$ y $\left.\Phi_{Y}=\tilde{\Phi}\right)$, we can summarize with the following diagram:

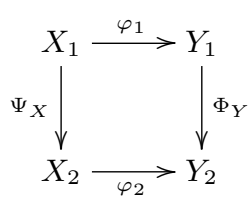

where $\Psi_{X}, \Phi_{Y}, \varphi_{1}$ y $\varphi_{2}$ are 1-block codes and $\Psi_{X}$ is right closing. By Proposition 17 , there exists a shift space $X_{1}^{\prime}$, a conjugacy $\Theta: X_{1}^{\prime} \rightarrow X_{1}$ and an u-right-resolving 1-block code $\Psi_{X}^{\prime}$ such that $\Psi_{X}^{\prime} \circ \Theta=\Psi_{X}$.

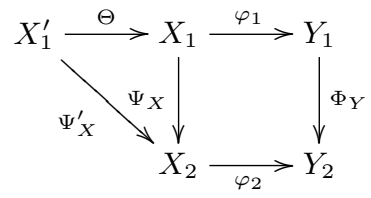

As $\Theta$ is a 1-block code, $\varphi_{1}^{\prime}=\varphi_{1} \circ \Theta$ is as well. Then, symplifying, we have a commuting diagram involving only 1-block codes and such that $\Psi_{X}^{\prime}, \varphi_{1}^{\prime}$ and $\varphi_{2}$ are e-right-resolving (because $\Psi_{X}^{\prime}$ is u-right-resolving and it can be verified $\varphi_{1}^{\prime}$ and $\varphi_{2}$ are e-right-resolving codes noting that $X$ and $Y$ are full-shifts) between irreducible SFT.

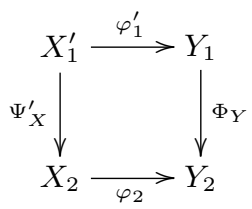

Claim: $\Phi_{Y}$ is e-right-resolving. In fact, let $y_{1}^{2} y_{2}^{2} \in \mathcal{L}\left(Y_{2}\right)$ and $y_{1}^{2} \in \mathrm{A}\left(Y_{1}\right)$ be such that $\phi_{Y}\left(y_{1}^{1}\right)=y_{1}^{2}$. As $\varphi_{1}$ is surjective (because it is a factor), there exists $x_{1}^{1} \in \mathrm{A}\left(X_{1}\right)$ such that $\varphi_{1}\left(x_{1}^{1}\right)=y_{1}^{1}$. Then, by commutativity of the diagram, $y_{1}^{2}=\phi_{Y} \circ \varphi_{1}\left(x_{1}^{1}\right)=\varphi_{2} \circ \psi_{X}\left(x_{1}^{1}\right)$. On the other hand, it is easy to verify that the composition of two e-right-resolving 1-block codes is e-right-resolving as well. Next, we have that $y_{1}^{2} y_{2}^{2} \in \mathcal{L}\left(Y_{2}\right)$ and $\varphi_{2} \circ \psi_{X}\left(x_{1}^{1}\right)=$ $x_{1}^{1}$, and, by the e-right-resolving property of $\varphi_{2} \circ \psi_{X}$, there exists $x_{2}^{1} \in \mathrm{A}\left(X_{1}\right)$ such that $x_{1}^{1} x_{2}^{1} \in \mathcal{L}\left(X_{1}\right)$ and $\varphi_{2} \circ \psi_{X}\left(x_{1}^{1} x_{2}^{1}\right)=y_{1}^{2} y_{2}^{2}$. Considering $y_{2}^{1}=\varphi_{2}\left(x_{2}^{1}\right)$, the e-right-resolving property of $\Phi_{X}$ follows.

Finally, as $\Phi$ is surjective (because $\Psi$ is right closing and $\Phi$ is a quotient of $\Psi$ ) and there are only conjugacies involved, we have that $h\left(Y_{1}\right)=h\left(Y_{2}\right)$, and, by Lemma 4 . $\Phi_{X}$ is u-right-resolving. As $\Phi_{X}$ and $\Phi$ are conjugated, $\Phi$ is right closing, for a sliding block code is right closing if and only if it is conjugated to an u-right-resolving 1-block code. 